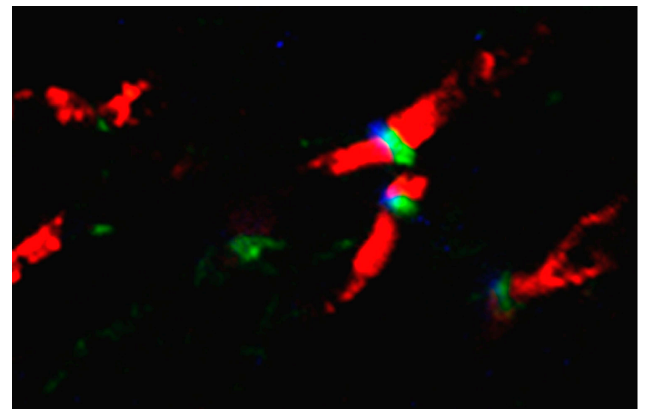

Antibodies (green) against neurofascin bind to the unmyelinated nodes of Ranvier (blue) in axons (red).

\section{Axon-attacking antibodies}

Axons have an Achilles' heel that antibodies can attack to cause multiple sclerosis (MS), Mathey et al. show on page 2363.

MS is triggered when $\mathrm{T}$ cells breach the blood-brain barrier and produce inflammatory cytokines that activate myelin-scavenging macrophages, causing damage to the myelin sheath. Myelin-specific antibodies that enter the brain in the T cells' wake enhance the destruction of the sheath and exacerbate disease. Researchers have blamed this myelin destruction for the neurological problems of patients with MS. But in some patients, damage happens not just to the myelin, but to the axons themselves. Because myelin can be replaced, axon damage is now considered to be the cause of permanent disability.

Mathey et al. now find that some MS patients have antibodies that attack the parts of axons known as the nodes of Ranvier. These nodes must be myelin free to allow clusters of voltage-gated ion channels to propagate action potentials.

The newly identified antibodies recognized a structural node protein called neurofascin-186 (NF-186). The antibodies interrupted neurotransmission in tissue sections and worsened disease symptoms by damaging axons in a rat model of MS.

Higher levels of these antibodies were found in patients who have a particularly degenerative version of the disease. The study included only a small number of patients, but if the trend continues in larger groups, the authors will then test whether getting rid of antibody-producing $\mathrm{B}$ cells or filtering the antibodies out of the patients' blood might halt disease progression. JEM

\section{T cells raise the tension}

The price of immunity might be high blood pressure, as data from Guzik et al. (page 2449) suggest.

High blood pressure-or hypertension-is triggered by angiotensin II. This hormone instructs the kidneys to retain salt and the brain to release stress hormones. It also activates oxidases that generate free radicals, which narrow blood vessels and cause plaques to grow and harden the arteries.

Recent evidence suggests that angiotensin II might also get the immune system into the tension-raising act. Patients with immune systems weakened by immunosuppressive drugs or HIV infection have low blood pressure, which can skyrocket when they go off the drugs or start taking antiviral medications. One set of lymphocytes, the T cells, are the likeliest culprits, as they bear angiotensin II receptors and the same dangerous free radical-generating enzymes.

Guzik et al. now find that angiotensin II activates T cells to raise blood pressure. Mice that lack lymphocytes failed to develop hypertension after angiotensin II injection unless T cells were put back in. The hormone, they found, increased the T cells' ability to attach to and infiltrate blood vessel walls. The cells congregated in the underlying fatty tissue and secreted the inflammatory cytokine tumor necrosis factor (TNF), which further activates free radical production in the surrounding cells. Blocking TNF blunted hypertension.

The authors propose that T cells infiltrating the kidney and the brain might also amplify angiotensin II's damaging effects in these organs. JEM

\section{Buttoned-up vessels}

A study by Baluk et al. (page 2349) reveals how the organization of junctional proteins might keep endothelial cells in lymphatic vessels buttoned up but still allow fluid and cells to enter.

Lymph flows through a network that starts out as tiny vessels and then widens into collecting ducts. But how lymph gets from the blood into lymph vessels through the endothelial barrier is unclear. One model suggests that endothelial cells lack junctions and thus glide apart under mechanical stress. Another proposes that junctions break down to permit fluid entry and then reseal.

Baluk et al. did not favor either idea, as junctions are required to maintain vessel structure, and their breakdown and reconstruction spell excessive wear and tear on the vessel. The team traced the expression of junctional proteins in lymphatic vessels in mouse airways and found that the proteins formed continuous zipper-like structures in collecting ducts. In the tiny vessel entryways, however, they were organized into button-like clumps at the corners of adjacent endothelial cells.

The flaps between the buttons probably allow fluid to enter, but the authors are not sure whether cells also crawl in through these openings. In the lymph networks of mice whose airways were inflamed, leukocytes sidled alongside the buttoned openings rather than the zippered ends. But the team is yet to catch cells in the act of creeping through. JEM
A junctional protein (red) clumps (arrows) at lymph vessel entryways but is continuous (arrowheads) in the lower collecting ducts.

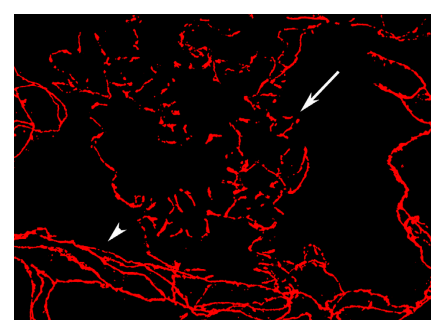

